\section{Clinical features of recently diagnosed papillary thyroid carcinoma in elderly patients aged 65 and older based on 10 years of sonographic experience at a single institution in Korea}

\author{
Eun Sil Kim', Younghen Lee', Hyungsuk Seo', Gil Soo Son², Soon Young Kwon³, \\ Young-Sik Kim ${ }^{4}$, Ji-A Seo ${ }^{5}$, Nan Hee Kim ${ }^{5}$, Sang-il Suh ${ }^{6}$, Inseon Ryoo ${ }^{6}$, Sung-Hye You ${ }^{7}$ \\ Departments of ${ }^{1}$ Radiology, ${ }^{2}$ Surgery, ${ }^{3}$ Otolaryngology-Head and Neck Surgery, and ${ }^{4}$ Pathology, \\ ${ }^{5}$ Division of Endocrinology, Department of Internal Medicine, Korea University Ansan Hospital, \\ Korea University College of Medicine, Ansan; ${ }^{6}$ Department of Radiology, Korea University \\ Guro Hospital, Korea University College of Medicine, Seoul; ' Department of Radiology, Korea \\ University Anam Hospital, Korea University College of Medicine, Seoul, Korea
}

Purpose: The aim of this study was to assess the characteristics of papillary thyroid carcinoma (PTC) in patients aged 65 and older in order to predict postoperative recurrence based on the results of ultrasonographic surveillance.

Methods: Among 1,494 patients (200 male and 1,294 female; mean age, 46.6 \pm 11.3 years) who underwent surgery for thyroid cancer at our institution between 2006 and 2015, we retrospectively enrolled 150 PTC patients ( 29 male and 121 female; mean age, $69.4 \pm 4.2$ years) aged 65 years and older. To identify the risk factors for recurrence, we analyzed age, gender, multiplicity, size, number, extrathyroidal extension (ETE) of the tumor, lymph node metastasis (LNM), type of surgery, and the dose of radioactive ablation using a Cox regression model to identify hazard ratios (HRs).

Results: Among the 115 asymptomatic patients with PTCs detected by screening ultrasonography $(n=86)$, other cross-sectional imaging modalities (computed tomography or positron emission tomography-computed tomography, $n=13)$, or incidentally through a surgical specimen $(n=16)$, 78 patients were confirmed to have papillary thyroid microcarcinomas (PTMCs). The other 35 patients presented with palpable neck masses $(n=25)$, vocal cord palsy $(n=9)$ or bloodtinged sputum $(n=1)$. During the follow-up period (mean, 43.6 months), 17 patients (12.5\%) experienced recurrence in the neck. None of the patients died due to PTC-related recurrence or distant metastasis during the follow-up period. Cox regression analysis demonstrated that tumor size $(H R, 2.12 ; P<0.001)$ and LNM (central LNM: HR, 9.08; $P=0.004$; lateral LNM: HR, 14.71; $P=0.002$; both central and lateral $L N M$ : $H R, 58.41 ; P<0.001)$ significantly increased the recurrence rate. ETE, LNM, and recurrence were significantly less frequent in PTMCs than in nonPTMC (all P<0.001).

Conclusion: PTCs of small size and absent LNM showed significantly better prognoses in patients 65 years and older.

Keywords: Thyroid cancer, papillary; Papillary thyroid microcarcinoma; Aged; Treatment outcomes; Surgery

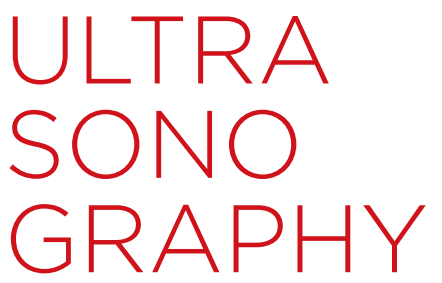

ORIGINAL ARTICLE

https://doi.org/10.14366/usg. 17010 pISSN: 2288-5919 • elSSN: 2288-5943 Ultrasonography 2017:36:355-362

Received: February 8, 2017

Revised: April 10, 2017

Accepted: April 13, 2017

Correspondence to:

Younghen Lee, MD, Department of Radiology, Korea University Ansan Hospital, Korea University College of Medicine, 123 Jeokgeum-ro, Danwongu, Ansan 15355, Korea

Tel. +82-31-412-5228

Fax. +82-31-412-5224

E-mail: younghen@korea.ac.kr

This is an Open Access article distributed under the terms of the Creative Commons Attribution NonCommercial License (http://creativecommons.org/ licenses/by-nc/3.0/) which permits unrestricted noncommercial use, distribution, and reproduction in any medium, provided the original work is properly cited.

Copyright $(2017$ Korean Society of Ultrasound in Medicine (KSUM)

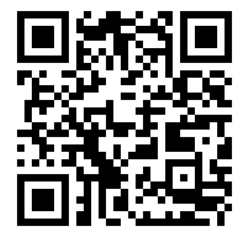

How to cite this article:

Kim ES, Lee Y, Seo H, Son GS, Kwon SY, Kim YS, et al. Clinical features of recently diagnosed papillary thyroid carcinoma in elderly patients aged 65 and older based on 10 years of sonographic experience at a single institution in Korea. Ultrasonography. 2017 Oct;36(4): 355-362. 


\section{Introduction}

Over the last decade, the incidence of differentiated thyroid cancers (DTCs), including papillary thyroid carcinoma (PTC) and follicular thyroid carcinoma, has increased worldwide [1-3]. Of particular note, in Korea, the annual incidence of thyroid cancer rose from 6.3 cases per 100,000 in 1999 to 52.7 cases per 100,000 in 2010, which represents a $25 \%$ annual increase over the last 10 years [4]. According to the United States population-based data from the Surveillance, Epidemiology, and End Results program from 2006 to 2012, PTC was most frequently diagnosed in patients aged 45-54 years, and approximately $20 \%$ of cases of thyroid cancer occurred in patients older than 65 years [5]. In the 2015 Korean Central Cancer Registry, thyroid cancer was reported to be the fourth most common cancer in women aged 65 years or older [6]. Despite its slow progression and highly favorable prognosis, PTC tends to show more aggressive histologic features, a greater tumor size, extensive local growth, and more frequent distant metastasis in elderly patients than in younger patients, leading to a poor prognosis with increased risks of recurrence and thyroid cancer-related mortality [7-16]. Therefore, the surgical treatment of thyroid cancer, such as total thyroidectomy and subsequent lifelong thyroid hormone replacement therapy, has inevitably become more common in the elderly based on the poor outcomes associated with advanced age, although most cases of PTC that have been recently diagnosed after the widespread implementation of screening ultrasonography (US) have been reported to be papillary thyroid microcarcinoma (PTMC), measuring $1 \mathrm{~cm}$ or less $[1,3,17,18]$. Some studies have advocated total thyroidectomy for elderly patients with PTC $[7,8,14,19,20]$. However, concerns have been raised about the possibility of postoperative subclinical thyrotoxicosis induced by thyrotropin suppression therapy, which can have cardiovascular effects or cause significant bone loss, particularly in older women [21,22].

To our knowledge, few studies have been conducted to support treatment decision-making in elderly patients with PTC $[7,8,11,15,19,21]$. In order to obtain reliable data on the initial diagnosis and postsurgical outcome of recently diagnosed PTC, patients' clinical features should be obtained from an institution where regular sonographic evaluations have been included in postoperative surveillance to detect small sized local recurrence. In addition, to understand the recently increased diagnosis of PTC, it would be helpful to review institutional records in Korea, where the incidence of PTC (age-standardized rate, 58.3\% per 100,000 population) has been reported to be more than 15 times greater than that of the UK and 5.6 times that of the United States, according to GLOBOCAN 2012 [23]. Herein, we reviewed the characteristics of patients aged 65 years and older who underwent surgery for PTC at a single institution and were subsequently followed by ultrasonography during the past 10 years to facilitate the prediction of recurrence.

\section{Materials and Methods}

\section{Patients}

We retrospectively reviewed the medical data of all patients who underwent surgery for thyroid cancer at our institution during the last 10 years, from January 2006 to December 2015. Of these 1,494 patients (200 male, 1,294 female; mean age: $46.6 \pm 11.3$ years), 153 patients were identified as being aged 65 years and older at the time of the initial diagnosis. Among the histologic types, PTC (150 patients) was the most common type, followed by follicular carcinomas (two patients) and malignant lymphoma (one patient). Ultimately, 150 patients aged 65 years and older (10.0\% of the total 1,494 patients) with PTC were enrolled in this study. This study was approved by the Institutional Review Board and the requirement for informed consent was waived.

\section{Preoperative Assessment}

In our institution, two board-certified radiologists with 15 and 17 years of experience in thyroid radiology, respectively, performed USguided fine needle aspiration (FNA) for suspected thyroid nodules and lymph nodes in the lateral compartment before surgery [24-26]. Each thyroid nodule was imaged in the transverse and longitudinal planes using the standard US protocol. Preoperative US images over the entire neck region were available in 125 of the 150 patients with pathologically proven PTCs. One board-certified radiologist with 15 years of experience in thyroid radiology who was aware of the pathologic results retrospectively reclassified the sonographic findings of PTC in 125 patients, according to the Korean Thyroid Image Reporting and Data System (K-TIRADS) criteria that were recently proposed by Shin et al. [26].

\section{Treatment}

All patients underwent macroscopically complete resection of the diagnosed PTC. In our hospital, total thyroidectomy was preferentially performed in patients older than 45 years, with multiple tumors, extrathyroidal extension (ETE), or lateral neck node metastasis on the preoperative assessment. Ipsilateral lobectomy was performed in patients with a tumor restricted to a single lobe and with no evidence of malignant nodules in the contralateral lobe. Therapeutic lateral neck dissection (LND) was performed only if lymph node metastasis (LNM) was preoperatively suspected. Prophylactic central neck dissection (CND) was generally performed on the ipsilateral or bilateral sides of the central compartment 
according to our institutional protocol. All surgical specimens of thyroids and lymph nodes were carefully reviewed by the pathologists.

In our hospital, most patients who underwent total thyroidectomy were treated by radioactive iodine therapy (RAIT) using different doses of ${ }^{131}$ I based on the patient's age, tumor size, lymph node status, and individual histological characteristics, and subsequently received thyrotropin suppression therapy.

\section{Post-treatment Follow-up}

All patients underwent regular follow-up at 6- to 12-month intervals with physical examinations, neck US and chest computed tomography, ${ }^{131}$ I scans, and serum thyroglobulin (Tg) measurements [24]. When suspicious lesions were detected, one of the two boardcertified radiologists with 15 and 17 years of experience in thyroid radiology performed US-guided FNA with Tg measurements [24]. The diagnosis of recurrence in the operative area in the thyroid or neck lymph node was made by the cytological documentation of malignant cells, an elevated Tg level in a FNA specimen, or pathologic diagnosis at the time of reoperation. The follow-up period for each patient was defined as the length of time between the initial operation until the last neck US and cross-sectional imaging [24].

\section{Variables}

The following data were obtained from the patients' medical records to identify the predictive factors of recurrence in thyroidectomized patients: initial presentation at diagnosis, initial US findings, pathologic results of the surgical specimen (size, multiplicity, and number of PTC; extent of ETE; and LNM), type of surgery, dose of RAIT, and postoperative surveillance results (local recurrence and distant metastasis).

Based on the pathologic results, tumor size was described as the largest diameter (for multiple lesions, including bilateral lesions, the maximum diameter of the largest lesion was used), and multiplicity was defined as the presence of more than one focus of PTC in either the ipsilateral or contralateral lobes. Patients were categorized as having PTMC if the size of the largest tumor was $1 \mathrm{~cm}$ or less; otherwise, they were classified as having non-PTMC. ETE was considered positive in the presence of a minimal extension of the PTC to perithyroidal soft tissue or sternothyroid muscle (equivalent to pathologic stage T3) or an extensive extension to the subcutaneous soft tissue, laryngotracheal airway, esophagus, or recurrent laryngeal nerve (equivalent to pathologic stage T4) [27]. Patients were classified as positive for LNM if PTC was present in the lymph nodes on a histologic examination. The central compartment lymph nodes (cervical level VI) equivalent to pathologic stage N1a included any of the following: the pretracheal, paratracheal, and prelaryngeal lymph nodes. The lateral lymph nodes equivalent to pathologic stage N1b included any of the following: unilateral, bilateral, or contralateral cervical level I-V lymph nodes [27].

\section{Statistical Analysis}

First, to identify the variables that were independently associated with postoperative recurrence, we performed multivariate regression analysis using a Cox proportional hazards model by estimating the respective hazard ratios (HRs) and 95\% confidence intervals (Cls). Covariates included age, gender, size, multiplicity, type of surgery, dose of RAIT, extent of ETE (minimal or extensive), and LNM (central, lateral, or both). Patients with incomplete data or those lost to follow-up were excluded from this retrospective analysis. Second, to assess the significant difference between PTMC and non-PTMC, the variables were compared using the Student $t$ test (continuous variables), the chi-square test, or the Fisher exact test (categorical variables). Continuous variables were expressed as mean \pm standard deviation, and categorical variables as number and percentage. $P$-values of $\leq 0.05$ were considered to indicate statistical significance. All calculations were performed using the SPSS ver. 20.0 (IBM Corp., Armonk, NY, USA).

\section{Results}

\section{Patient Characteristics and Primary Tumor Features at Initial Surgery}

The age at diagnosis of the 150 elderly patients aged 65 years or older ranged from 65 to 86 years (mean, $69.4 \pm 4.2$ years). Among them, $121(80.7 \%)$ were female and 29 were male. Regarding the type of surgery, total thyroidectomy with CND was most commonly performed, in 110 patients. Hemithyroidectomy without CND, hemithyroidectomy with CND, total thyroidectomy with CND and unilateral LND, and total thyroidectomy with CND and bilateral LND were performed in six, 21, nine, and four patients, respectively. The patients who underwent total thyroidectomy received RAIT with dosages of $30 \mathrm{mCi}(n=5,3.3 \%), 100 \mathrm{mCi}(n=36,24.0 \%), 150 \mathrm{mCi}$ $(n=41,27.3 \%)$, or $200 \mathrm{mCi}(n=1,1.0 \%)$. RAIT was not performed in low-risk patients with a single tumor with a size of $1 \mathrm{~cm}$ or less, or in those who underwent hemithyroidectomy ( $n=67,44.7 \%)$.

At the initial presentation, asymptomatic PTCs were preoperatively suspected in 115 patients by US-guided FNA after screening US $(n=86)$ or other cross-sectional imaging such as computed tomography $(n=10)$ or positron emission tomography-computed tomography ( $n=3)$, and the other 16 PTCs were incidentally identified after surgery for benign thyroid disease. In contrast, the PTCs in the remaining 35 patients were symptomatic at the initial 
presentation. The most frequent symptoms were palpable neck masses $(n=25)$; other initial presentations were vocal cord palsy in nine patients and blood-tinged sputum in one patient. The most common histologic subtype was conventional type $(n=140)$, while the remaining 10 PTCs were confirmed to be the follicular variant $(n=8)$, the mixed tall cell and solid variant $(n=1)$, and the oncocytic variant $(n=1)$, respectively.

Preoperative US images were available for 125 patients, including images obtained from other hospitals. Based on the K-TIRADS criteria [26], the PTCs of 76 patients were category 5 (high suspicion), while category 4 (intermediate suspicion) and category 3 (low suspicion) findings were seen in 33 and three patients, respectively. The PTCs of the other 13 patients could not be classified because they were not clearly delineated on their preoperative sonographic examinations.

The mean size of the surgical specimens of PTCs was $1.4 \pm 1.4 \mathrm{~cm}$ (range, 0.1 to $7.0 \mathrm{~cm}$ ) in the greatest dimension, and 83 patients had PTMCs measuring $\leq 1 \mathrm{~cm}$. The PTMCs of 16 patients were unsuspected prior to surgery. The number of pathologically proven PTCs was $1.5 \pm 0.7$ (range, 1 to 4), and multiplicity was present in 53 patients. Among the 58 patients (38.7\%) with evidence of ETE, most showed minimal extension to the perithyroidal soft tissue $(n=50)$, while the other eight patients showed wide extension, such as to the trachea, larynx, or recurrent laryngeal nerve.

Of the 150 patients, lymph node dissection was performed in $144(96.0 \%)$. Sixty-three patients showed LNM in the central compartment $(n=52)$, lateral compartment $(n=6)$, or both the central and lateral compartments $(n=5)$.

Based on the postoperative surveillance data, the mean followup period was $43.5 \pm 30.0$ months (range, 6 to 100 months), and none of the enrolled patients died due to PTC-related recurrence or distant metastasis during the follow-up period. Fourteen patients (9.3\%) were lost to periodic follow-up after the initial operation. During the follow-up period, 17 patients (12.5\%) experienced recurrence in the lateral neck $(n=13)$ or the operative area in the thyroid $(n=4)$, as detected by US-guided FNA and Tg studies. The mean time from initial treatment to recurrence in these patients was $43.8 \pm 28.1$ months. These patients were treated by surgical resection. No patient experienced secondary recurrence during the follow-up period.

\section{Predictive Factors of Recurrence in Elderly Patients with PTC}

Multivariate Cox regression analysis revealed that only tumor

Table 1. Multivariate analysis of the postoperative recurrence of PTC in elderly patients who were periodically examined after the operation

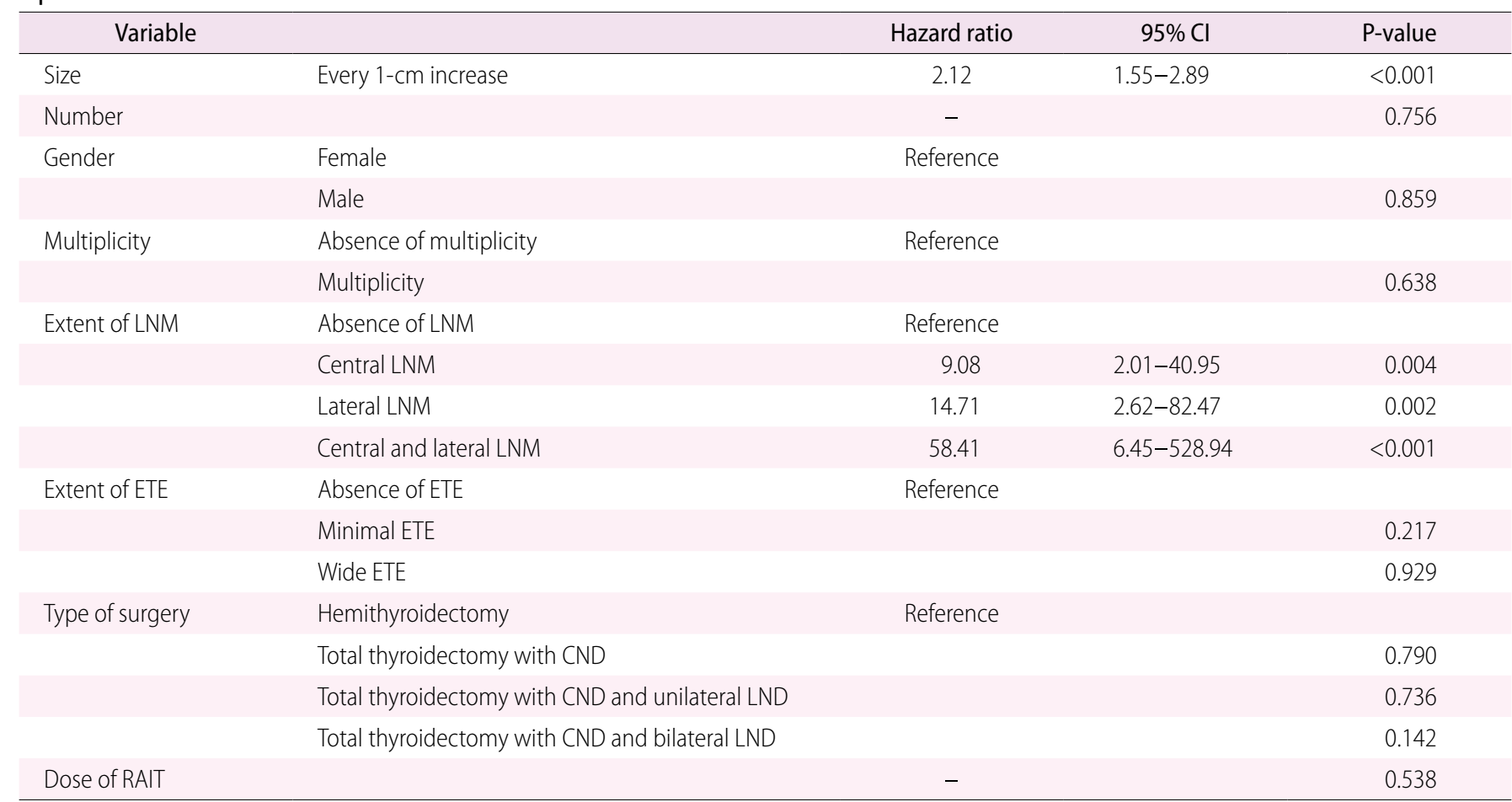

PTC, papillary thyroid carcinoma; Cl, confidence interval; LNM, lymph node metastasis; ETE, extrathyroidal extension; CND, central neck dissection; LND, lateral neck dissection; RAIT, radioactive iodine therapy. 
Table 2. Clinical features of PTMC and non-PTMC

\begin{tabular}{|c|c|c|c|}
\hline Variable & PTMC $(\leq 1 \mathrm{~cm}, \mathrm{n}=83)$ & Non-PTMC (>1 cm, n=67) & P-value \\
\hline Age (yr) & $68.7 \pm 3.3$ & $70.2 \pm 4.9$ & 0.025 \\
\hline Female sex & $66(79.5)$ & $55(82.1)$ & 0.836 \\
\hline Size $(\mathrm{cm})$ & $0.6 \pm 0.2$ & $2.3 \pm 1.6$ & $<0.001$ \\
\hline Number & $1.4 \pm 0.7$ & $1.5 \pm 0.8$ & 0.472 \\
\hline Multiplicity & $29(34.9)$ & $24(35.8)$ & $>0.990$ \\
\hline Total thyroidectomy & $64(77.1)$ & $59(88.1)$ & 0.127 \\
\hline RAIT (\%) & $30(36.1)$ & $53(79.1)$ & $<0.001$ \\
\hline Dose of RAIT (mCi) & $37.6 \pm 54.8$ & $104.2 \pm 59.8$ & $<0.001$ \\
\hline ETE & $15(25.9)$ & $43(74.1)$ & $<0.001$ \\
\hline Minimal extension & $15(25.9)$ & $35(52.2)$ & 0.014 \\
\hline Wide invasion & 0 & $8(11.9)$ & $<0.001$ \\
\hline LNM & $23(27.7)$ & 40 (59.7) & $<0.001$ \\
\hline Central LNM & $23(27.7)$ & $35(52.2)$ & 0.003 \\
\hline Lateral LNM & $4(4.8)$ & $8(11.9)$ & 0.195 \\
\hline Recurrence & $2(2.7)$ & $15(24.2)$ & $<0.001$ \\
\hline Follow-up period (mo) & $43.5 \pm 28.1$ & $43.6 \pm 29.4$ & 0.985 \\
\hline
\end{tabular}

Values are presented as mean \pm SD or number (\%).

PTMC, papillary thyroid microcarcinoma (largest diameter of the tumor $\leq 1 \mathrm{~cm}$ ); non-PTMC, non-papillary thyroid microcarcinoma (largest diameter of the tumor $>1 \mathrm{~cm}$ ); RAIT, radioactive iodine therapy; ETE, extrathyroidal extension; LNM, lymph node metastasis.

size and the extent of LNM were significant risk factors for the postoperative recurrence of recently diagnosed PTCs in elderly patients (Table 1). With each 1-cm increment in tumor size, the risk of recurrence increased 2.12-fold ( $95 \% \mathrm{Cl}, 1.55$ to 2.89; $\mathrm{P}<0.001)$. Regarding the extent of LNM, the risk of recurrence was 9.08fold higher with central LNM ( $95 \% \mathrm{Cl}, 2.01$ to 40.95), 14.71-fold higher with lateral LNM $(95 \% \mathrm{Cl}, 2.62$ to 82.47$)$ and 58.41 -fold higher with both central and lateral LNM $(95 \% \mathrm{Cl}, 6.45$ to 528.94$)$, compared to those without pathologically proven $L N M(P=0.004$, $P=0.002$, and $P<0.001$, respectively). Other factors, such as age, gender, tumor number, multiplicity, type of surgery, the dose of RAIT, and extent of ETE (minimal or extensive), were not significantly associated with recurrence (all $\mathrm{P}>0.05$ ).

\section{Comparison of PTMC and Non-PTMC at Initial Presentation and Postoperative Surveillance}

Based on the pathologic reports, 83 patients with tumors measuring $\leq 1 \mathrm{~cm}$ were classified as having PTMC, and the other 67 patients with tumors measuring $>1 \mathrm{~cm}$ were classified as having non-PTMC. The size of the PTCs and the mean age of patients significantly differed between the two groups $(0.6 \pm 0.2 \mathrm{~cm}$ vs. $2.3 \pm 1.6 \mathrm{~cm}, P<0.001$; $68.7 \pm 3.3$ years vs. $70.2 \pm 4.9$ years, $P=0.025)$, but no significant difference was found in gender or in the percentage of total thyroidectomy ( $P>0.05$ ) (Table 2). The frequency of ETE, specifically for both minimal and wide extension, was significantly higher in the non-PTMC group than in the PTMC group; in addition, the non-PTMC group showed a higher rate of LNM (both $P<0.001$ ). In contrast, there was no significant difference in multiplicity between the two groups ( $34.9 \%$ vs. $35.8 \%, P>0.990)$. The recurrence rate for PTMC was significantly lower than that for non-PTMC $(P<0.001)$. Distant metastasis was not detected in any of the patients.

\section{Discussion}

Unlike in other malignancies, the patient's age has been regarded as an independent risk factor for PTC [10-14]. However, the reported data are insufficient to specifically address PTC recently diagnosed in elderly patients. Most staging systems have chosen the cutoff of 45 years of age to differentiate low-risk DTC from highrisk DTC $[16,28,29]$. Moreover, various cutoff ages, such as 60,65 , 75 , or 85 years, have been used to evaluate the clinical features of elderly patients due to the lack of a precise definition of "old age" $[7,8,10,11,13-15,19]$. Therefore, the actual proportion of elderly patients who have been reported to undergo elective thyroid surgery ranges from $2.5 \%$ to $21.2 \%$, depending on the criteria used for patient selection.

To the best of our knowledge, this is the first study to reflect the recently increased diagnosis of asymptomatic PTMC in elderly 
patients, although the overall survival was not fully evaluated because of the relatively short follow-up period. Based on initial and serial sonographic surveillance in the past 10 years, 83 patients (55.3\%) were diagnosed with PTMC among the 150 patients aged 65 and older with PTC, and the overall rate of recurrence was $12.5 \%$ (17 of 139) among patients who were followed up for a mean of 43.3 months.

In this study, tumor size and LNM were identified as independent predictors of recurrence in elderly patients with PTC, which is consistent with previous studies that reported that size, local extent ( $\mathrm{T}$ staging) and nodal status ( $\mathrm{N}$ staging) were important prognostic factors in patients 45 years or older $[16,20,28-30]$. In addition, the HR of LNM was greater than that of tumor size (more than 9-, 14-, and 58-fold higher with LNM in the central, lateral, and both central and lateral compartments, respectively, than in those without any LNM, as compared to a twofold increase with each 1-cm increment in tumor size), indicating that LNM may be more closely associated with recurrence in the elderly than tumor size. Since US is widely recognized to be the preoperative imaging modality of choice to assess LNM in cases of PTC [24-26], the diagnostic role of preoperative US cannot be overemphasized for elderly patients with PTC.

PTMC has been considered to be an indolent disease due to its low risk of recurrence and high overall survival $[31,32]$. The recurrence rate of our elderly patients with PTMC was $2.7 \%$, which is similar to the result of a recent 11-year observation of PTMC patients from 2000 to 2011, which revealed that only six of 202 patients (mean age, 49 years; range, 15 to 83 years) experienced recurrence after surgery, with a median time to recurrence of approximately 12 months [33]. These results are also compatible with those of a 10-year observational study of 1,235 patients with low-risk PTMC that was conducted by Ito et al. [31]. They emphasized the presence of significant age-specific differences in tumor biology between subclinical PTMC and clinically advanced PTC; for example, the incidence of larger tumors, LNM, and progression to clinical disease was lowest in oldest patients ( $\geq 60$ years) and highest in the youngest patients ( $<40$ years) [31]. At the time of their initial diagnosis, our elderly patients with PTMC likewise showed significantly fewer cases of ETE and LNM, which led to a lower postoperative recurrence rate than was observed among those with non-PTMC, even with a relatively short followup interval. Accordingly, elderly patients with subclinical PTMC have been recommended as candidates for observation rather than immediate surgery $[31,34]$. In another study, Ito et al. [35] concluded that thyroid surgery is not always necessary unless a patient exhibits LNM, multiplicity, a positive family history, or other pathological lesions in the contralateral lobe.
Our results differ from those of previous studies that recruited elderly patients with PTC in several aspects. First, a relatively high percentage $(42.3 \%)$ of elderly patients underwent surgical resection for asymptomatic PTMCs. This is related to the unique medical environment in Korea. Since 2002, thyroid US has become an optional workup in health promotion programs at most hospitals in Korea, meaning that people can easily undergo US screenings at a cost of $\$ 30-\$ 50$, which has led to an abrupt increase in the detection and treatment of PTC $[3,18]$. For this reason, the frequency of thyroid cancer diagnoses has increased in Korea 15fold over the past 20 years $[6,18]$; however, the mortality rate has remained almost constant during the past 3 decades $[3,17,18]$. Even in the elderly population, the majority of newly diagnosed cases of thyroid cancer in Koreans are small PTCs that infrequently exhibit LNM or ETE [3]. Second, the recurrence rate found in this study $(12.5 \%)$ is less than one-third of the rate of $39 \%$ that was obtained in a study with a larger population of 2,883 patients over 65 years of age who were diagnosed with DTC between 1995 and 2007 [15]. This difference may be explicable in part based on the availability of detailed preoperative US examinations. Currently, prior to surgery, experienced radiologists carefully examine the primary thyroid tumor and the adjacent neck lymph nodes, like in our hospital; therefore, postoperative recurrence has markedly declined, irrespective of the patient's age at initial presentation [24-26]. On the contrary, preoperative US was not routinely performed during the early period of that study (from 1995 to 2007). Therefore, the higher recurrence rates in the previous studies can be explained by the initially missed nonpalpable lymph nodes, which became clinically evident years after surgery. For similar reasons, age was not regarded as an independent predictor of recurrence in a recently published observational study of a Chinese cohort of 407 PTC patients, regardless of whether age was entered as a categorical or continuous variable, as in this study [13].

Due to the recent rapid increase in the life expectancy of the general population, the elderly population has increased by $90 \%$ over the last 30 years. Based on the 2010 United States Census, by 2030, more than $20 \%$ of United States residents are projected to be aged 65 and over, compared with 13\% in 2010 and $9.8 \%$ in 1970, in addition, the population aged 65 and over is projected to be 83.7 million in 2050, almost double the corresponding figure of 43.1 million in 2012 [36]. Unlike the younger population, elderly patients often have concomitant medical problems, some of which may be potentially lethal conditions such as cardiovascular, respiratory, or renal disease. If they undergo total thyroidectomy for thyroid cancer, there is a small but significant risk of operative complications, such as vocal cord paralysis or hypoparathyroidism, and lifelong thyroid hormone replacement may be needed [21]. Based on our 10-year 
single-institution experience, we advocate a tailored approach to develop appropriate treatment or follow-up plans in elderly patients depending on the tumor size and the extent of ETE or LNM $[2,21,34]$. More aggressive treatment (total thyroidectomy and subsequent RAIT) or more frequent follow-up should be considered only for patients with a larger tumor, wide ETE, or lateral LNM, all of which can be predicted by preoperative radiologic assessment. In contrast, a more conservative management strategy, including active surveillance rather than immediate surgery, would be preferred for elderly patients with PTMC, which shows less frequent ETE or LNM, as in a recently published risk stratification [37].

Nonetheless, our study has several limitations. First, given the indolence of PTC, including PTMC, this study was conducted over a relatively short follow-up period (mean, 43.3 months; range, 6 to 100 months); therefore, it was not possible to fully assess the impact of the characteristics of PTC on late recurrence and late death. Second, to elucidate the prognostic factors of recently diagnosed PTC in elderly patients, we enrolled a small number of elderly patients with PTC treated at a single institution. A larger populationbased prospective study should be performed in various regions and hospital settings with a longer follow-up period to support our results. Third, because of the retrospective design of this study, it was not possible to adjust for some confounding factors, such as the surgeon's preference for thyroid surgery for elderly patients, so some patients diagnosed with PTMC underwent total thyroidectomy, especially during the earlier period.

In conclusion, PTCs of small size and absent LNM showed significantly better prognoses in patients 65 years and older. Therefore, a tailored treatment approach should be established for elderly patients with PTC, in which the treatment strategy can be primarily determined by the patients' US findings.

ORCID: Eun Sil Kim: http://orcid.org/0000-0002-0632-9902; Younghen Lee: http://orcid.org/0000-0001-7739-4173; Hyungsuk Seo: http://orcid.org/00000003-2303-7415; Gil Soo Son: http://orcid.org/0000-0001-8684-7875; Soon Young Kwon: http://orcid.org/0000-0002-1501-390X; Young-Sik Kim: http://orcid.org/00000001-9681-572X; Ji-A Seo: http://orcid.org/0000-0002-1927-2618; Sang-il Suh: http://orcid.org/0000-0001-8933-0492; Inseon Ryoo: http://orcid.org/0000-00016866-7926; Sung-Hye You: http://orcid.org/0000-0001-5005-9383

\section{Conflict of Interest}

No potential conflict of interest relevant to this article was reported.

\section{References}

1. Brito JP, Morris JC, Montori VM. Thyroid cancer: zealous imaging has increased detection and treatment of low risk tumours. BMJ 2013;347:f4706.

2. O'Grady TJ, Gates MA, Boscoe FP. Thyroid cancer incidence attributable to overdiagnosis in the United States 1981-2011. Int J Cancer 2015;137:2664-2673.

3. Cho BY, Choi HS, Park YJ, Lim JA, Ahn HY, Lee EK, et al. Changes in the clinicopathological characteristics and outcomes of thyroid cancer in Korea over the past four decades. Thyroid 2013;23:797804.

4. Jung KW, Won YJ, Kong HJ, Oh CM, Lee DH, Lee JS. Cancer statistics in Korea: incidence, mortality, survival, and prevalence in 2011. Cancer Res Treat 2014:46:109-123.

5. National Cancer Institute. Surveillance, Epidemiology, and End Results Program. SEER Stat Fact Sheets: thyroid cancer. Bethesda, MD: National Cancer Institute, 2016.

6. Jung KW, Won YJ, Oh CM, Kong HJ, Cho H, Lee DH, et al. Prediction of cancer incidence and mortality in Korea, 2015. Cancer Res Treat 2015;47:142-148.

7. Calo PG, Medas F, Loi G, Erdas E, Pisano G, Nicolosi A. Differentiated thyroid cancer in the elderly: our experience. Int J Surg 2014;12 Suppl 2:S140-S143.

8. Park HS, Jung CK, Lee SH, Chae BJ, Lim DJ, Park WC, et al. Clinicopathologic characteristics and surgical outcomes of elderly patients with thyroid cancer. Jpn J Clin Oncol 2014;44:1045-1051.

9. Park HS, Roman SA, Sosa JA. Treatment patterns of aging Americans with differentiated thyroid cancer. Cancer 2010;116:2030.

10. Coburn MC, Wanebo HJ. Age correlates with increased frequency of high risk factors in elderly patients with thyroid cancer. Am J Surg 1995; 170:471-475.

11. Vini L, Hyer SL, Marshall J, A'Hern R, Harmer C. Long-term results in elderly patients with differentiated thyroid carcinoma. Cancer 2003; $97: 2736-2742$.

12. Eustatia-Rutten CF, Corssmit EP, Biermasz NR, Pereira AM, Romijn JA, Smit JW. Survival and death causes in differentiated thyroid carcinoma. J Clin Endocrinol Metab 2006;91:313-319.

13. Lang BH, Lo CY, Wong KP, Wan KY. Long-term outcomes for older patients with papillary thyroid carcinoma: should another age cutoff beyond 45 years be added? Ann Surg Oncol 2015;22:446453.

14. Longheu A, Medas F, Pisano G, Gordini L, Nicolosi A, Sorrenti $S$, et al. Differentiated thyroid cancer in patients $\geq 75$ years: histopathological features and results of surgical treatment. Int J Surg 2016;33 Suppl 1:S159-S163.

15. Hollenbeak CS, Boltz MM, Schaefer EW, Saunders BD, Goldenberg D. Recurrence of differentiated thyroid cancer in the elderly. Eur J Endocrinol 2013;168:549-556.

16. Nixon IJ, Wang LY, Palmer FL, Tuttle RM, Shaha AR, Shah JP, et al. The impact of nodal status on outcome in older patients with papillary thyroid cancer. Surgery 2014;156:137-146. 
17. Ahn HS, Kim HJ, Kim KH, Lee YS, Han SJ, Kim Y, et al. Thyroid cancer screening in South Korea increases detection of papillary cancers with no impact on other subtypes or thyroid cancer mortality. Thyroid 2016;26:1535-1540.

18. Ahn HS, Kim HJ, Welch HG. Korea's thyroid-cancer "epidemic": screening and overdiagnosis. N Engl J Med 2014;371:1765-1767.

19. Marvin K, Parham K. Differentiated thyroid cancer in people aged 85 and older. J Am Geriatr Soc 2015;63:932-937.

20. de Castro TP, Waissmann W, Simoes TC, de Mello RC, Carvalho DP. Predictors for papillary thyroid cancer persistence and recurrence: a retrospective analysis with a 10-year follow-up cohort study. Clin Endocrinol (Oxf) 2016;85:466-474.

21. McLeod DS, Carruthers K, Kevat DA. Optimal differentiated thyroid cancer management in the elderly. Drugs Aging 2015;32:283-294.

22. Wang LY, Smith AW, Palmer FL, Tuttle RM, Mahrous A, Nixon IJ, et al. Thyrotropin suppression increases the risk of osteoporosis without decreasing recurrence in ATA low- and intermediate-risk patients with differentiated thyroid carcinoma. Thyroid 2015;25:300-307.

23. International Agency for Research on Cancer. Glossary of terms from cancer incidence in five continents volume $X(C \mid 5 X)$ [Internet]. Lyon: IARC, 2013 [cited 2017 Mar 2]. Available from: http://ci5.iarc. fr/Cl5plus/Pages/glossary.aspx.

24. Haugen BR, Alexander EK, Bible KC, Doherty GM, Mandel SJ, Nikiforov YE, et al. 2015 American Thyroid Association management guidelines for adult patients with thyroid nodules and differentiated thyroid cancer: the American Thyroid Association Guidelines Task Force on Thyroid Nodules and Differentiated Thyroid Cancer. Thyroid 2016;26:1-133.

25. Yeh MW, Bauer AJ, Bernet VA, Ferris RL, Loevner LA, Mandel SJ, et al. American Thyroid Association statement on preoperative imaging for thyroid cancer surgery. Thyroid 2015;25:3-14.

26. Shin JH, Baek JH, Chung J, Ha EJ, Kim JH, Lee YH, et al. Ultrasonography diagnosis and imaging-based management of thyroid nodules: revised Korean Society of Thyroid Radiology consensus statement and recommendations. Korean J Radiol 2016;17:370-395.

27. Edge SB, Byrd DR, Compton CC, Fritz AG, Greene FL, Trotti A. AJCC cancer staging manual. 7th ed. New York: Springer, 2010;87-96.
28. Zaydfudim V, Feurer ID, Griffin MR, Phay JE. The impact of lymph node involvement on survival in patients with papillary and follicular thyroid carcinoma. Surgery 2008;144:1070-1077.

29. Tran Cao HS, Johnston LE, Chang DC, Bouvet M. A critical analysis of the American Joint Committee on Cancer (AJCC) staging system for differentiated thyroid carcinoma in young patients on the basis of the Surveillance, Epidemiology, and End Results (SEER) registry. Surgery 2012;152:145-151.

30. Amin MB, Edge $S$, Greene F, Byrd DR, Brookland RK, Washington MK, et al. AJCC cancer staging manual. 8th ed. New York: Springer, 2017.

31. Ito $Y$, Miyauchi A, Kihara M, Higashiyama T, Kobayashi K, Miya A. Patient age is significantly related to the progression of papillary microcarcinoma of the thyroid under observation. Thyroid 2014;24:27-34.

32. Sugitani I, Toda K, Yamada K, Yamamoto N, Ikenaga M, Fujimoto Y. Three distinctly different kinds of papillary thyroid microcarcinoma should be recognized: our treatment strategies and outcomes. World J Surg 2010;34:1222-1231.

33. Siddiqui $S$, White MG, Antic T, Grogan RH, Angelos P, Kaplan EL, et al. Clinical and pathologic predictors of lymph node metastasis and recurrence in papillary thyroid microcarcinoma. Thyroid 2016;26:807-815.

34. Shakil J, Ansari MZ, Brady J, Xu J, Robbins RJ. Lower rates of residual/recurrent disease in patients with incidentally discovered thyroid carcinoma. Endocr Pract 2017;23:163-169.

35. Ito Y, Kudo T, Kobayashi K, Miya A, Ichihara K, Miyauchi A. Prognostic factors for recurrence of papillary thyroid carcinoma in the lymph nodes, lung, and bone: analysis of 5,768 patients with average 10-year follow-up. World I Surg 2012;36:1274-1278.

36. Ortman JM, Velkoff VA, Hogan H. Current population reports. An aging nation: the older population in the United States. Washington, DC: U.S. Census Bureau, 2014;25-1140.

37. Brito JP, Ito $Y$, Miyauchi $A$, Tuttle RM. A clinical framework to facilitate risk stratification when considering an active surveillance alternative to immediate biopsy and surgery in papillary microcarcinoma. Thyroid 2016;26:144-149. 